\title{
Selecting a Theoretical Framework to Guide a Research Study of Older Adults' Perceptions and Experiences of Falling in the Hospital
}

Hanne Dolan, MSN, RN, PhD Student

The University of Arizona

College of Nursing

1305 N Martin Ave, Tucson, AZ 85721-0203

hannedolan@email.arizona.edu

Ruth Taylor-Piliae, PhD, RN, FAHA

The University of Arizona

College of Nursing

1305 N Martin Ave, Tucson, AZ 85721-0203

rtaylor@nursing.arizona.edu

Corresponding Author: Hanne Dolan, MSN, RN, PhD Student, The University of Arizona College of Nursing, 1305 N Martin Ave, Tucson, AZ 85721-0203, hannedolan@email.arizona.edu 


\begin{abstract}
A paucity of research has explored the older adult's experience of falling in the hospital. Understanding the central concepts associated with a fall while hospitalized is essential for further fall prevention research and practice. The purpose of this paper is to describe the process of selecting a theoretical framework to guide a qualitative study exploring the older adult's experience of falling while hospitalized. An analysis of six established illness self-management theories and models from nursing and psychology was conducted using Walker and Avant's framework for theory analysis. The Health Belief Model was selected as the most appropriate theoretical framework, as it entails concepts applicable to the experience of falling and captures the complexity of the phenomenon of inpatient falls, which is important for nursing.
\end{abstract}

Keywords: Heath Belief Model, inpatient falls, older adult, theoretical framework. 


\section{Introduction}

Patient falls in acute care is a persistent problem and limited evidence was reported in support of any single preventative intervention to reduce inpatient fall rates (Cameron et al., 2012). Inpatient falls has been explored empirically with quantitative research studies. However, fall rates in hospitals have not significantly changed (Agency for Health Research and Quality, 2019). On the other hand, qualitative studies of community dwelling older adults' perception of falling and being labeled at risk for falling, found the experience to be embarrassing and shameful, with a loss of independence and dignity, preventing the older adult from adhering to fall prevention measures (Hallrup, Albertsson, Tops, Dahlberg, \& Grahn, 2009; McInnes, Seers, $\&$ Tutton, 2011). This paper presents the process of selecting a theoretical framework for a qualitative study exploring the older adult's experience of falling in the hospital.

\section{Frameworks and Models for Understanding Illness Self-Management}

Inpatient falls is a complex phenomenon important to nursing. Connecting a theoretical framework to the problem of inpatient falls is essential in order to understand the older adult's perspective and promote self-management of this problem. Theories and models from both nursing and psychology were evaluated for relevance to the falling phenomenon using Walker and Avant's (2011) six-step method of theory analysis (i.e., origins, meaning, logical adequacy, usefulness, generalizability and parsimony, and testability).

\section{Nursing Theoretical Frameworks}

Dorothea Orem's Self-Care Deficit Nursing Theory (SCNDT) is a grand theory with abstract concepts that applies to global nursing phenomena, by defining what nursing is and who is in need of nursing (Denyes, Orem, \& Bekel, 2001). The SCNDT consists of the theory of selfcare, the theory of self-care deficit and the theory of nursing systems. These middle range 
theories have defined concepts, propositions and empirical indicators foundational for nursing research and practice (Biggs, 2008; Denyes et al., 2001). The individual's self-care system is the performed actions that regulate normal life development and functioning. When faced with an illness, new self-care demands emerge and if the individual is unable to meet the self-care demands, they develop self-care deficits with the individual needing nursing care (Denyes et al., 2001). Orem's SCNDT can apply to inpatient falls, since being at risk for falling is a self-care demand, and the inability to manage one's own fall risk is a self-care deficit. However, the SCNDT does not focus on the individual's health beliefs and possible barriers that prevent the older adult from accepting a self-care deficit. The SCNDT is thereby not well suited for exploring the experience of falling in the hospital.

The middle-range Theory of Self-Care in Chronic Illness (TSCCI) aims to understand the individual's process of performing self-care and maintaining health during management of chronic illness (Riegel, Jaarsma, \& Strömberg, 2012). The TSCCI is strongly influenced by Orem's SCNDT, yet Riegel and colleagues (2012) developed the theory inductively from their collective experience working with heart failure patients. The main concepts of the TSCCI are self-care maintenance, self-care monitoring and self-care management, representing the process of self-care in chronic illness (Jaarsma, Cameron, Riegel, \& Stromberg, 2017). The concepts and assumptions in the TSCCI are well defined, and the theory includes testable propositions (Riegel et al., 2012). Decision-making and reflection is a part of the self-care process, and the TSCCI applies to individuals with normal cognitive function and with long-term chronic illness (Jaarsma et al., 2017). The TSCCI can apply to fall prevention when falls are a symptom of a chronic illness. However, the theory may not adequately capture the phenomenon of falling in the 
hospital, since falling in this setting not only occurs among individuals with chronic illnesses, but also among older adults who are acutely ill and cognitively impaired.

The Self-Help Model (SHM) developed by Dr. Braden seeks to frame the experience of living with a chronic illness (Braden, 1990). The main underlying assumption is that learning is a fundamental process in human beings and chronic illness presents a new learning frame (Braden, 1991). The SHM was developed deductively from pre-existing learning theories and inductively from prior working experience with chronically ill individuals (Braden, 1990). The key concept of perceived severity of the illness affects how individuals are able to provide self-help in managing their chronic illness. Uncertainty and dependency negatively affect self-help, whereas enabling skills and resourcefulness positively affects self-help leading to better quality of life (Braden, 1990, 1991). The SHM posits logical adequacy, usefulness, parsimony and generalizability, and the model has been utilized as a research framework and empirically tested (Braden, 1990). Falling presents uncertainty and possible dependency, and falling requires selfhelp and management of one's own fall risk. The SHM can inform the phenomenon of inpatient falls, however, the model does not explicitly focus on the barriers that prevent acceptance of one's own fall risk or learning how to manage one's own fall risk when hospitalized.

\section{Theoretical Frameworks From Psychology}

Dr. Leventhal's Common-Sense Model of Self-regulation (CSM) examines behavioral, cognitive and perceptual processes among individuals' self-management of current and future health threats (Leventhal, Phillips, \& Burns, 2016). The CSM was developed through empirical research over several decades, and the model has clearly defined assumptions, concepts and propositions. The CSM describes the dynamic process of how illness threats are represented and managed. When the normal functioning self is threatened by a condition, such as a fall, the self 
must regulate itself to maintain homeostasis. The CSM can promote understanding of the individual's problem-solving mechanisms that are initiated when an illness threat emerges (Leventhal et al., 2016). The CSM is a well-established model that could be applied to inpatient falls, since a fall threatens the older adult's normal functioning of self. While the model may explain the older adult's actions against the threat of falling, the CSM does not specifically focus on barriers to fall risk acceptance in acutely ill hospitalized older adults.

In Bandura's Social Cognitive Theory (SCT), self-efficacy is understood as the individual's judgement of their capability of certain actions that guides a specific behavior (Bandura, 1977; Resnick, 2014). Performance accomplishments, emotional arousal, vicarious experience and verbal persuasion influence expectations of self-efficacy. The SCT contains the triadic reciprocality with the person-behavior-environment interaction that is the foundation for human function (Resnick, 2014). Bandura's SCT has been thoroughly tested over several decades and can explain and predict psychological changes in an individual when presented with aversive experiences (Bandura, 1977). The SCT can apply to inpatient fall prevention by focusing on the older adults' self-efficacy in managing their fall risk. However, self-efficacy requires adequate perceived confidence in reducing one's fall risk, which the hospitalized older adult may be lacking due to acute illness exacerbations impacting their fall risk.

Finally, the Health Belief Model (HBM) was developed in the 1950's in an attempt to understand why people did not participate in screenings and preventative health strategies for detection of asymptomatic disease (Janz \& Becker, 1984). The HBM was developed by social psychologists and evolved deductively from learning theories and inductively from practical public health concerns (Champion \& Skinner, 2008). The central concepts are centered around the individual's perception of an illness threat and what initiates action or not towards an illness 
threat. The concepts and conceptual relationships are clearly stated (Rosenstock, 1974).

Perceived susceptibility is understood as the individuals' acceptance of susceptibility to a condition ranging from denial to fear of contracting the condition and perceived severity is the individual's convictions about the seriousness of the disease (Rosenstock, 1974). Perceived benefits refers to the individuals' acceptance of their susceptibility and benefits from actions, such as medical treatment (Janz \& Becker, 1984). Perceived barriers are the potential negative aspects of a health action, such as unpleasant side-effects or embarrassment. The barriers can be conflicting and lead to avoidance (Rosenstock, 1974). Cues to action are the internal and external triggers that lead to decision-making, while self-efficacy is the individual's perceived ability to perform an intended action in the disease process (Janz \& Becker, 1984; Rosenstock, Strecher, \& Becker, 1988). The HBM has been utilized in research for decades confirming the usefulness, generalizability and testability of the model (Champion \& Skinner, 2008; Janz \& Becker, 1984). The HBM applies to the phenomenon of falling in the hospital, since the model is not limited to chronic illness or preventative behavior. The HBM can capture the broad range of emotions and reactions associated with falling or being at risk for falling in the hospital. The model applies to older hospitalized adults who may not yet understand or accept their own susceptibility to falling due to acute illness. As well, the HBM applies to hospitalized older adults who understand their own fall risk, are afraid of falling, and are considering perceived barriers and benefits associated with fall prevention. Therefore, the HBM was selected as the best theoretical framework for a qualitative research study exploring older adults' experience of falling in the hospital.

\section{The Health Belief Model and the Experience of Falling}

The experience of falling and being labeled at risk for falling is associated with emotions such as embarrassment, loss of independence and denial of one's own fall risk. These emotional 
consequences can prevent the older adult from adhering to fall prevention interventions, such as asking for assistance or using a walker (Gettens, Fulbrook, Jessup, \& Low Choy, 2018; Hallrup, Albertsson, Bengtsson Tops, Dahlberg, \& Grahn, 2009; McInnes et al., 2011). Gettens et al. (2018) found that some older hospitalized adults did not perceive themselves as at risk for falling, since the nursing staff would keep them safe, in spite of the older adult already having suffered a fall in the hospital. The older adults' perceived susceptibility to and perceived severity of falling affect what actions they take to avoid falling. Embarrassment and fear of embarrassment can be barriers to fall prevention efforts and may hinder any perceived benefits of fall prevention (McInnes et al., 2011; Rosenstock, 1974). Fear of embarrassment may also be a cue to action, since suffering a fall in front of others can be embarrassing. Avoiding this situation is strongly desired (Babcock, 1988). At this point, fall prevention may have more perceived benefits than barriers. Understanding hospitalized older adults' perceived susceptibility to falling, and their perceived barriers and benefits of fall prevention, is essential in the effort to reduce falls among hospitalized older adults. Theory-guided research into this problem is needed.

\section{Conclusion}

Analyzing and critiquing illness self-management theories and models to discover a framework that adequately captures the nursing phenomenon of interest, is an essential step in the research process. The HBM provides a comprehensive framework for guiding a qualitative research study exploring older adults' experiences of falling in the hospital. The HBM can encompass a wide range of emotions and reactions experienced by hospitalized older adults, and also addresses the benefits and barriers of fall risk acceptance and fall prevention adherence. Not only is the model useful for research in this area but it has relevance to nursing practice and may potentially lead to reduced inpatient fall rates. 


\section{References}

Agency for Healthcare Research and Quality (2019). National scorecard on hospital-acquired conditions updated baseline rates and preliminary results 2014-2017. Retrieved on 9/32019 from https://www.ahrq.gov/sites/default/files/wysiwyg/professionals/qualitypatient-safety/pfp/hacreport-2019.pdf

Babcock, M. K. (1988). Embarrassment: A window on the self. Journal for the Theory of Social Behaviour, 18(4), 459-483. http://dx.doi.org/10.1111/j.1468-5914.1988.tb00510.x

Bandura, A. (1977). Self-efficacy: Toward a unifying theory of behavioral change. Psychological Review, 84(2), 191-215. doi:10.1037/0033-295X.84.2.191

Biggs, A. (2008). Orem's self-care deficit nursing theory: Update on the state of the art and science. Nursing Science Quarterly, 21(3), 200-206. doi:10.1177/0894318408320140

Braden, C. J. (1990). A test of the self-help model: Learned response to chronic illness experience. Nursing Research, 39(1), 42-47.

Braden, C. J. (1991). Patterns of change over time in learned response to chronic illness among participants in a systemic lupus erythematosus self-help course. Arthritis \& Rheumatism: Official Journal of the American College of Rheumatology, 4(4), 158-167.

Cameron, I., Gillespie, L., Robertson, C., Murray, G., Hill, K., Cumming, R., \& Kerse, N. (2012). Interventions for preventing falls in older people in care facilities and hospitals. Cochrane Database of Systematic Reviews, 12, doi:10.1002/14651858.CD005465.pub2.

Champion, V. L., \& Skinner, C. S. (2008). The health belief model. In K. Glanz, B. K. Rimer, \& K. Viswanath (Eds.), Health behavior and health education: Theory, research, and practice (pp. 45-65). San Fransisco, CA: Jossey-Bass. 
Denyes, M. J., Orem, D. E., \& Bekel, G. (2001). Self-care: A foundational science. Nursing Science Quarterly, 14(1), 48-54. doi:10.1177/089431840101400113

Gettens, S., Fulbrook, P., Jessup, M., \& Low Choy, N. (2018). The patients' perspective of sustaining a fall in hospital: A qualitative study. Journal of Clinical Nursing, 27(3-4), 743-752. doi:10.1111/jocn. 14075

Hallrup, L. B., Albertsson, D., Bengtsson Tops, A., Dahlberg, K., \& Grahn, B. (2009). Elderly women's experiences of living with fall risk in a fragile body: A reflective lifeworld approach. Health \& Social Care in the Community, 17(4), 379-387. doi:10.1111/j.13652524.2008.00836.x

Jaarsma, T., Cameron, J., Riegel, B., \& Stromberg, A. (2017). Factors related to self-care in heart failure patients according to the middle-range theory of self-care of chronic illness: A literature update. Current Heart Failure Reports, 14(2), 71-77. doi:10.1007/s11897-0170324-1

Janz, N. K., \& Becker, M. H. (1984). The health belief model: A decade later. Health Education Quarterly, 11(1), 1-47. doi:10.1177/109019818401100101

Leventhal, H., Phillips, L. A., \& Burns, E. (2016). The Common-Sense Model of SelfRegulation (CSM): A dynamic framework for understanding illness self-management. Journal of Behavioral Medicine , 39(6), 935-946. doi:10.1007/s10865-016-9782-2

McInnes, E., Seers, K., \& Tutton, L. (2011). Older people's views in relation to risk of falling and need for intervention: A meta-ethnography. Journal of Advanced Nursing, 67(12), 2525-2536. doi:10.1111/j.1365-2648.2011.05707.x

Resnick, B. (2014). The theory of self-efficacy. In M. J. Smith \& P. Liehr (Eds.), Middle range theory for nursing (pp. 197-223): New York, NY: Springer Publishing Company. 
Riegel, B., Jaarsma, T., \& Strömberg, A. (2012). A middle-range theory of self-care of chronic illness. Advances in Nursing Science, 35(3), 194-204. doi:10.1097/ANS.0b013e318261b1ba.

Rosenstock, I. M. (1974). Historical origins of the health belief model. Health Education Monographs, 2(4), 328-335. https://doi.org/10.1177\%2F109019817400200403

Rosenstock, I. M., Strecher, V. J., \& Becker, M. H. (1988). Social learning theory and the health belief model. Health Education Quarterly, 15(2), 175-183. doi:10.1177/109019818801500203

Walker, L. O., \& Avant, K. C. (2011). Strategies for theory construction in nursing (5th ed.). Upper Saddle River, NJ: Prentice Hall. 